\title{
Supplementary Information Table S2.
}

Secondary structure at interface, $\mathrm{S}=$ sheet, $\mathrm{H}=$ helix and $\mathrm{C}=$ coil.

\begin{tabular}{|c|c|c|c|}
\multicolumn{1}{c}{} & \multicolumn{1}{c}{ PDB } & Target & Partner \\
\hline ZipA/FtsZ & $1 \mathrm{f} 47$ & $\mathbf{S}$ & $\mathbf{H}$ \\
\hline BcIXL/Bak & $1 \mathrm{bxI}$ & $\mathbf{H}$ & $\mathbf{H}$ \\
\hline MDM2/p53 & $1 \mathrm{ycr}$ & $\mathbf{H}$ & $\mathbf{H}$ \\
\hline MDM2/p53 & $1 \mathrm{ycq}$ & $\mathbf{H}$ & $\mathbf{H}$ \\
\hline XIAP BIR3/SMAC & $1 \mathrm{~g} 73$ & $\mathbf{S}$ & $\mathbf{S}$ \\
\hline XIAP BIR3/CASPASE 9 & $1 \mathrm{nw} 9$ & $\mathbf{S}$ & $\mathbf{S}$ \\
\hline
\end{tabular}

\begin{tabular}{|c|c|c|c|}
\multicolumn{1}{c}{} & \multicolumn{1}{c}{ PDB } & Target & Partner \\
\hline Subtilisin/Eglin C & $1 \mathrm{cse}$ & C & S \\
\hline Subtilisin/Eglin C & 1 rOr & C & C \\
\hline Subtilisin/Eglin C & 1 to 2 & C & S \\
\hline Trypsin/trypsin inh & 2 uuy & C & C \\
\hline Thrombin/Protein & $3 b 9 f$ & C & C \\
\hline HPV E2/HPV E1 & 1 tue & H & H \\
\hline Chagasin/papain & $3 e 1 z$ & C & C \\
\hline FKBP12/TGFR & $1 b 6 c$ & S & H \\
\hline MMP1/TIMP1 & $2 j 0 t$ & C & C \\
\hline MMP3/TIMP1 & 1009 & C & C \\
\hline IL-2/IL-2R & $1 z 92$ & H & S \\
\hline
\end{tabular}

